\title{
The tracking system of the IDEA detector concept for a future $\mathrm{e}^{+} \mathrm{e}^{-}$collider
}

\author{
Giovanni F. Tassielli ${ }^{a, b, 1, *}$ \\ a Dipartimento Interateneo di Fisica 'M. Merlin', Università degli studi di Bari \\ ${ }^{b}$ INFN Sezione di Bari, \\ via Amendola 173, 70125 Bari, Italy \\ E-mail: gianfranco.tassielli@cern.ch
}

The IDEA detector concept for future $\mathrm{e}^{+} \mathrm{e}^{-}$colliders proposes a tracking system composed by a Si based inner system, an ultra-low mass Drift Chamber central system with Particle Identification capabilities and a Si based outer layer surrounding the drift chamber. The designed tracking system allows to fulfill the high momentum and angular resolutions requirements for the whole momentum range, particularly for low momenta, thanks to the extremely low material budget. Moreover, the use of the Cluster Counting technique allows for particle identification (PID) resolution below 3\%, a factor two better than the resolution attainable with traditional $\mathrm{dE} / \mathrm{dx}$ techniques. Details about the construction of the drift chamber, including both the speculation about new materials for the field wires and new techniques for soldering the wires, the development of an improved layout of the drift cells, and the choice of the gas mixture will be described. The expected tracking system performance together with the Improved PID obtained with the cluster counting technique will be reported.

European Physical Society conference on high energy physics 2021 - EPS-HEP Conference 2021 Online conference, July 26 - July 30, 2021

\footnotetext{
${ }^{1}$ On behalf of the IDEA collaboration.

* Speaker
} 


\section{1. $\mathrm{FCC}-\mathrm{e}^{+} \mathrm{e}^{-}$and the tracking requirements}

The high-luminosity future circular electron-positron collider, FCC-ee [1], is designed to deliver a clean environment to study precisely the interactions with center-of-mass energies tunable at: the $\mathrm{Z}$ pole $(91 \mathrm{GeV})$, the WW threshold $(161 \mathrm{GeV})$, the Higgs factory $(240 \mathrm{GeV})$ and around the $t \bar{t}$ threshold (340-365 GeV). Respect to the linear collider options, the FCC-ee will provide much higher luminosity at low energy ( $\sim 3$ orders of magnitude higher at the $\mathrm{Z}$ pole) that decreases with energy reaching almost the same value around the top quark pair production threshold. Thus the FCC-ee will allow to study with high precision the Z, W, Higgs and top particles by producing clear samples of $5 \times 10^{12} \mathrm{Z}$ bosons, $10^{8} \mathrm{~W}$ pairs, $10^{6} \mathrm{Higgs}$ bosons and $10^{6}$ top quark pairs. These features have implications on the detector optimization, in particular on the tracking systems, since at low energy the multiple scattering contribution to the track parameter resolution becomes important, while at high energies the asymptotic terms are more relevant. Moreover, the extreme luminosity at the $\mathrm{Z}$ pole achievable generates a physics event rate of $\sim 100 \mathrm{kHz}$ imposing strict constraints on sub-detectors rate capability and on DAQ systems.

Synthetically, the constraints imposed by machine characteristics, interaction region layout and physics performance for the tracking systems they can be summarized as following:

- for the Central tracker:

- state-of-the-art momentum and angular resolution for charged particles $\left(\sigma\left(1 / p_{T}\right) \lesssim 3 \times 10^{-5}(\mathrm{GeV} / c)^{-1}\right.$ and $\sigma(\Theta, \phi) \sim 0.1 \mathrm{mrad}$ for $45 \mathrm{GeV}$ muons);

- to contain the vertical emittance at $\mathrm{Z}$ pole the $\mathrm{B}$ field is limited to $\sim 2 \mathrm{~T}$, this require a large tracking radius to recover momentum resolution;

- high transparency required given typical momenta in Z, H decays (far form the asymptotic limit where the Multiple Scattering contribution is negligible);

- Particle ID is a valuable additional ability;

- for the Vertex tracker:

- excellent b-and c-tagging capabilities : few $\mu \mathrm{m}$ precision for charged particle origin;

- small pitch, thin layers, limited cooling, first layer as close as possible to IP.

\section{The Innovative Detector for Electron-positron Accelerators detector concept}

The IDEA detector is a general purpose detector designed for experiments at future $\mathrm{e}^{+} \mathrm{e}^{-}$colliders. It is made by a silicon pixel vertex detector, a large-volume extremely light drift chamber surrounded by a layer of silicon micro-strip detectors, a thin and low-mass superconducting solenoid coil, a preshower detector, a dual-readout calorimeter, a muon system inside the magnet return yoke, more details can be found in $[1,2]$.

The vertex detector consists of a five layers of Si detectors, based on monolithic active pixels of $20 \mu \mathrm{m}$ size, that extend radially from $1.5 \mathrm{~cm}$ up to $\sim 35 \mathrm{~cm}$. It has to provide a space point resolution of $3 \mu \mathrm{m}$ in both directions to reach the requirement on the asymptotic track impact parameter resolution of $2 \mu \mathrm{m}$. The current design is based on the Si pixel detectors of the ALICE inner tracker system (ITS) upgrade.The results of the ALICE ITS upgrade, indicate an excellent ( $\lesssim 5 \mu \mathrm{m}$ ) resolution, high efficiency at low power consumption (power dissipation not exceeding 20 $\mathrm{mW} / \mathrm{cm}^{2}$ ) and very light detectors, $0.3-0.5 \% \mathrm{X}_{0}$ per layer. This would be a good starting point for 
the IDEA vertex detector, moreover it can profit of the new ongoing developments for Si sensors as the one in the context of the INFN ARCADIA R\&D project and of the AIDAinnova project founded by the European Commission (EC).

The central tracker is based on a drift chamber $(\mathrm{DCH})$ designed to provide good tracking, high-precision momentum measurement and excellent particle identification by using the cluster counting technique. It will be discussed in more details in the next paragraph.

A double layer of a Si micro-strips tracker will surround the DCH to provide an additional accurate space point ( $\sim 15 \mu \mathrm{m}$ on the transverse plane), as well as to improve the asymptotic momentun resolutiona and to precisely defining the tracker acceptance.

\section{The IDEA central drift chamber and the tracking system performance}

The Central Drift chamber ( $\mathrm{CDCH})$ is a unique-volume, high granularity, fully stereo, lowmass cylindrical drift chamber, co-axial with the $2 \mathrm{~T}$ solenoid field. It extends from an inner radius $R_{\text {in }}=0.35 \mathrm{~m}$ to an outer radius $R_{\text {out }}=2 \mathrm{~m}$, for a length $L=4 \mathrm{~m}$ and consists of 112 co-axial layers, at alternating-sign stereo angles, arranged in 24 identical azimuthal sectors. The stereo angles range from $50 \mathrm{mrad}$ to $250 \mathrm{mrad}$. The square cell size varies between $12.0 \mathrm{~mm}$ and $14.5 \mathrm{~mm}$ for a total of 56,448 drift cells. Each cell is designed with a ratio of field to sense wires equal to 5:1 to ensure the proper electrostatic configuration, and is composed by one anode and two cathode sub-layers, more details can be found in [2,3]. The anodes are $20 \mu \mathrm{m}$ diameter tungsten wires, while the cathodes are 40 and $50 \mu \mathrm{m}$ light aluminum alloy wires. In total, the $\mathrm{CDCH}$ is made with 56,448 sense wires, 285,504 cathode wires and 2,016 guard wires to equalize the gain of the innermost and outermost layers. The construction of the IDEA CDCH could be possible only by adopting the novel approach for the wiring and for the assembly procedures $[3,4]$ developed for the construction of the $\mathrm{CDCH}$ of MEG II experiment at PSI. However, the larger amount of the wires needed by the IDEA CDCH imposes severe limits on the wire breaking probability, for this reason R\&D programs are started to identify possible new material for the construction of the thin wires as alternative to the metallic ones used for MEG II. Under the EC projects AIDAinnova and CREMLIN+ R\&D programs are ongoing to coat thin carbon wires with small amount of metallic materials to make them electrically conductive and solderable.

The IDEA CDCH active volume is confined within a cylindrical carbon fiber shells at the outer and inner radius with equivalent thicknesses respectively of $1.2 \% \mathrm{X}_{0}$ and $0.08 \% \mathrm{X}_{0}$. The $\mathrm{CDCH}$ gas mixture is $90 / 10 \mathrm{He} / \mathrm{i}-\mathrm{C}_{4} \mathrm{H}_{10}$, chosen for the low radiation length $(\sim 1400 \mathrm{~m})$, a fast enough average drift velocity $(\sim 2 \mathrm{~cm} / \mu \mathrm{s})$ corresponding to a maximum drift time less than $400 \mathrm{~ns}$, and a good spatial resolution (with standard readout technique $\sim 110 \mu \mathrm{m},[5]$ ).

The momentum resolution of the IDEA tracking system was evaluated with a Geant 4 simulation and with an analytic approach. Assuming a single cell resolution of $100 \mu \mathrm{m}$ for the CDCH and conservative spatial resolution ( $\mathrm{pitch} / \sqrt{12}$ ) for Si detectors, the IDEA tracking system meets the expected performance. Moreover, the resolution at high momentum $(\sim 100 \mathrm{GeV} / c)$ can be improved (from $4 \times 10^{-5}$ to $2.9 \times 10^{-5}$ usign a less conservative spatial resolution for the Si detectors, Fig. 1-right). Due to the minimization of the multiple scattering contribution the IDEA tracking system performs better, over almost the entire momentum range of interest, than an alternative 

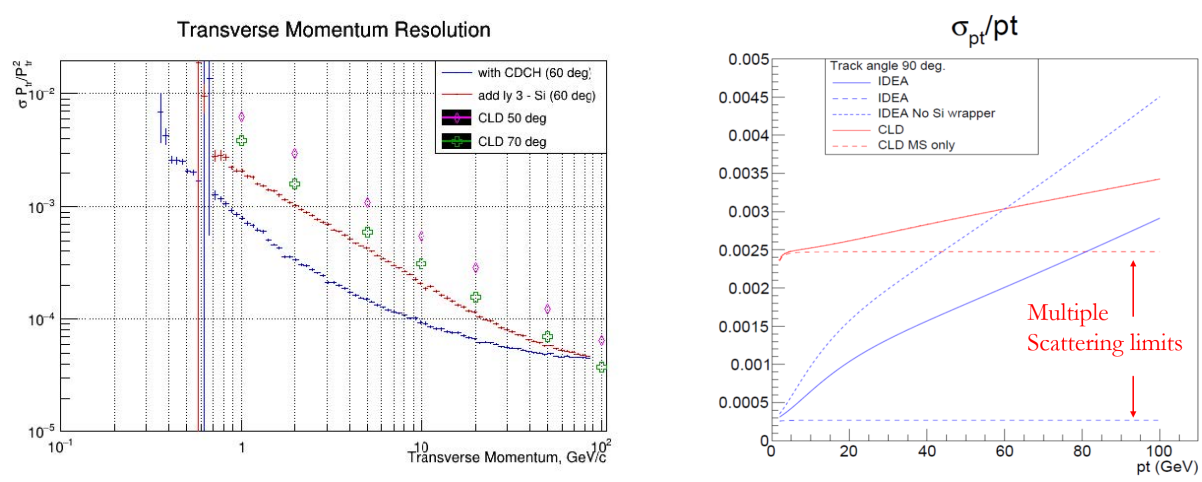

Figure 1: Conparison of the transverse momentum resolutions of the IDEA tracking system and of a full Si based tracking system similar to the one of the CLD detecor [1] as obtained with: Geant4 simulation (left), an analytic calculation of the full covariance matrixe that takes into account spatial resolution and material effects (right).

tracking system based only on Si detectors, see Fig. 1. Furthermore, $\mathrm{CDCH}$ will adopt the cluster counting and timing techniques, which allow for particle identification (PId) with unprecedented resolutions, where it is assumed that one can reach a relative resolution on the measurement of the number of primary ionisation clusters, $N_{c l}$, equal to $1 / \sqrt{N_{c l}}$. For the chosen gas, the number of ionisation clusters generated by a minimum ionizing particle (m.i.p.) is about $12.5 \mathrm{~cm}^{-1}$, allowing for efficiently exploiting the cluster counting/timing techniques to improve both spatial resolution $\left(\sigma_{r \phi}<100 \mu \mathrm{m}\right)$ and particle identification $\left(\sigma\left(d N_{c l} / d x\right) /\left(d N_{c l} / d x\right)<3.6 \%\right)$. The expected PId performance as a function of momentum, for $\mu / \pi, \pi / \mathrm{K}, \mathrm{K} / \mathrm{p}$, in terms of numbers of standard deviation was evaluated with analytical calculations and simulation. Assuming a cluster counting efficiency of $80 \%$, the cluster counting technique can guarantee gain of a factor 2 with respect to $\mathrm{dE} / \mathrm{dx}$ approach over almost the whole momentum range, more details are reported in [6].

\section{References}

[1] A. Abada et al., FCC-ee: The Lepton Collider, European Physical Journal Special Topics 228:2 261-623 (2019).

[2] G.F. Tassielli, A proposal of a drift chamber for the IDEA experiment for a future $e^{+} e^{-}$collider, PoS ICHEP2020 (2021) 877.

[3] G. F. Tassielli et al., The Drift Chamber of the MEG II experiment, JINST 15 C09051 (2020).

[4] G. Chiarello et al., The construction technique of the high granularity and high transparency drift chamber of MEG II, JINST 12 C07022 (2017).

[5] A. M. Baldini et al., Single-hit resolution measurement with MEG II drift chamber prototypes, JINST 11 P07011 (2016).

[6] F. Cuna et al., A proposal of a He based Drift Chamber as central tracker for the IDEA detector concept for a future $e^{+} e^{-}$collider, PoS EPS-HEP2021 (2021) 786. 